\title{
Size Determination of (Bio)conjugated Water-Soluble Colloidal Nanoparticles: A Comparison of Different Techniques
}

\author{
R. A. Sperling, ${ }^{\dagger}$ T. Liedl,${ }^{\dagger}$ S. Duhr, ${ }^{\dagger}$ S. Kudera, ${ }^{\dagger}$ M. Zanella, ${ }^{\dagger}$ C.-A. J. Lin,${ }^{\dagger}$, W. H. Chang, ${ }^{\dagger}$ \\ D. Braun, ${ }^{\dagger}$ and W. J. Parak $*, \dagger$ \\ Center for Nanoscience, Ludwig Maximilians Universität, München, Germany, and \\ Center for Nano Bioengineering and R\&D Center for Membrane Technology, \\ Chung Yuan Christian University, Taiwan, R.O.C.
}

Received: February 5, 2007; In Final Form: April 21, 2007

\begin{abstract}
The size of inorganic colloidal nanoparticles coated with organic layers of different thickness has been measured with different techniques, including transmission electron microscopy, gel electrophoresis, size exclusion chromatography, fluorescence correlation spectroscopy, and thermophoresis. The results are critically compared, and the advantages and disadvantages of the respective methods are discussed.
\end{abstract}

\section{Introduction}

In the past decades, improved synthesis techniques have influenced significantly research and applications of inorganic colloidal nanoparticles. This is mainly due to advances in the control of particle growth. Nowadays, samples with very narrow size distribution and controlled shape can be grown. ${ }^{1-3}$ Colloidal stability is provided by a layer of organic molecules around the inorganic particle core. This layer can be either hydrophobic or hydrophilic, and the respective particles are soluble in organic solvents or aqueous solution. Naturally, this layer contributes to the overall diameter of the particles. Whereas the colloidal stability of particles in organic solvents is usually achieved by a monolayer of hydrophobic molecules, ${ }^{4}$ thicker (hydrophilic) layers are often used to stabilize particles in aqueous solution, which in turn results in significantly increased particle diameters. ${ }^{5,6}$ The effective diameter of the particles increases further when additional (biological) molecules are bound to the particle surface in order to provide functionality. In particular, for biological applications, it is important to know the effective diameter of the particles, as bigger particles, for example, might not be able to enter pores of a certain size. However, due to the composite nature of the particles - a "hard" inorganic core and a "soft" organic shell with attached biological molecules-this is not a trivial task. The problem is that some techniques are more sensitive for the inorganic part, and others may eventually influence the conformation and thus the size of the organic part.

The size of the first generation of colloidal semiconductor nanoparticles was investigated extensively several years ago by the group of Henglein and Weller. ${ }^{7-9}$ These particles were directly synthesized in water, and the organic shell around the inorganic cores comprised just a monolayer of mercaptocarbonic acid molecules. In this study, we want to investigate the size of more complex nanoparticles that first have been synthesized in organic solvents, then have been transferred to aqueous solution by embedding them in a hydrophilic polymer shell, ${ }^{10-13}$ and finally have been modified by the conjugation of polyethylene glycol (PEG) molecules with different molecular weight. ${ }^{14}$ These

\footnotetext{
* Corresponding author. E-mail: Wolfgang.Parak@physik.uni-muenchen. de.

Ludwig Maximilians Universität.

Chung Yuan Christian University.
}

particles represent the general case where the samples differ in the thickness of a (soft) organic shell, while the (hard) inorganic particle core is the same.

For this work, nanoparticle size was characterized by the following methods: transmission electron microscopy (TEM), ${ }^{15-17}$ gel electrophoresis, ${ }^{9,18-24}$ size exclusion chromatography (SEC), ${ }^{7,8,21,25-35}$ fluorescence correlation spectroscopy (FCS), ${ }^{11,36-44}$ and thermophoresis. ${ }^{45-47}$ These and other relevant methods not used in this study are described in more detail in the Supporting Information (SI).

\section{Materials and Methods}

Particle Synthesis. CdSe/ZnS core/shell nanoparticles were synthesized in organic solvent according to standard protocols ${ }^{48,49}$ and transferred to aqueous solution by embedding them in a shell of an amphiphilic polymer. ${ }^{11}$ All protocols are reported in detail in the Supporting Information (SI §I.1-§I.5). The first exciton peak in the absorption spectrum of the CdSe cores was at $610 \mathrm{~nm}$ (corresponding to a diameter of the inorganic core of $4.7 \mathrm{~nm}^{50}$ ) and, after overcoating with the $\mathrm{ZnS}$ shell, at $614 \mathrm{~nm}$ (see Table 1). PEG molecules of different molecular weight, which were modified with an amino group on one end, were attached at different coverages by standard EDC chemistry to the polymer shell around the nanoparticles ${ }^{14}$ (SI §I.6). Sketches of the resulting particles are drawn in Tables 1-3. For particles whose surfaces were saturated with PEG, unbound excess PEG molecules were removed in five subsequent purification steps with centrifuge filters. Mixtures of particles with a discrete number of PEG molecules attached per particle were first run on $1 \%$ agarose gels in order to separate particles with zero, one, two, and three PEG molecules attached per particle. After extraction of the particles from the gel, they were purified on a desalting column. As additional samples, $\mathrm{Au}$ nanoparticles ${ }^{16,51}$ with the same modifications as those described for the $\mathrm{CdSe} / \mathrm{ZnS}$ particles were also used. Detailed protocols have been published previously. ${ }^{11,14} \mathrm{We}$ also measured the diameter of commercially available quantum dots with and without streptavidin modification (Table 4).

Transmission Electron Microscopy. TEM images of CdSe/ $\mathrm{ZnS}$ particles dissolved in chloroform and water were recorded before and after embedding them in a shell of amphiphilic 
TABLE 1: The Inorganic Hard Core Diameter $<d>$ and the Effective Diameters $<d_{\text {eff }}>$ of CdSe, CdSe/ZnS, and Au Particles (Drawn in Gray) Determined with Optical Methods and by TEM Images

\begin{tabular}{lcccc} 
particle & $\begin{array}{c}<>_{\text {abs }} \\
\text { type }\end{array}$ & $\begin{array}{c}<>_{\text {TEM }} \\
{[\mathrm{nm}]^{a}}\end{array}$ & $\begin{array}{c}<d_{\text {eff }}>_{\text {TEM }}[\mathrm{nm}] \\
\text { before polymer } \\
\text { coating }\end{array}$ & $\begin{array}{c}<d_{\text {eff }}>\text { TEM }[\mathrm{nm}] \\
\text { after polymer } \\
\text { coating }^{c}\end{array}$ \\
\hline $\mathrm{CdSe}$ & 5.1 & 4.7 & 6.0 & \\
$\mathrm{CdSe} / \mathrm{ZnS}$ & 5.3 & 5.3 & 6.4 & 9.6 \\
$\mathrm{Au}$ & & 4.6 & 5.6 & 9.0
\end{tabular}

${ }^{a}$ For the CdSe particles, the diameter was derived from absorption spectra. ${ }^{50}{ }^{b}$ By analyzing the spacing between the particles in the TEM images, the effective diameters $\left\langle d_{\text {eff }}>\right.$, which comprise the inorganic particles plus the organic layers (drawn in red and blue) attached to their surfaces, were determined before and after coating the particles with an amphiphilic polymer. Before the polymer coating, the particle is just surrounded by the hydrophobic surfactant layer (drawn in red). After the polymer coating, an amphiphilic polymer (with hydrophobic tails drawn in red and a hydrophilic backbone drawn in blue) is also wrapped around the particles. ${ }^{11,14}{ }^{c}$ These values have to be considered as an approximation (see SI §II.2).

polymer. Drops of the particle solution were placed on TEM grids, and images were recorded after evaporation of the solvent. The distribution of the inorganic particle diameters and the distances between the centers of adjacent particles were derived from the images by an image analysis program (SI §II.1).

Gel Electrophoresis. CdSe/ZnS and Au particles saturated with PEG molecules of different length were run on agarose gels $(1-2 \%, 1-2 \mathrm{~h}, 100 \mathrm{~V})$. As a control, phosphine-stabilized $10 \mathrm{~nm} \mathrm{Au}$ particles were also run on the same gel. ${ }^{19,20}$ After running the particles on the gel, the bands of the $\mathrm{CdSe} / \mathrm{ZnS}$ and $\mathrm{Au}$ particles were identified by their fluorescence and red color, respectively (see Figure 1) (SI §III). The mobilities of the different particles were determined from their position on the gel relative to the position where they had been loaded to the gel. ${ }^{19,20}$ For $\mathrm{CdSe} / \mathrm{ZnS}$ and $\mathrm{Au}$ particles with a discrete number of PEG molecules attached per particle, a low amount of PEG molecules of different length was reacted to the polymercoated $\mathrm{CdSe} / \mathrm{ZnS}$ and $\mathrm{Au}$ particles, and the reaction mixtures were run on agarose gels. After running the gel, discrete bands corresponding to $\mathrm{CdSe} / \mathrm{ZnS}$ and $\mathrm{Au}$ particles with no, exactly one, exactly two, and exactly three PEG molecules bound per particle were observed as individual bands on the gel (see Figure 1). ${ }^{19,20}$ The bands were extracted from the gel, and the obtained $\mathrm{CdSe} / \mathrm{ZnS}-$ and $\mathrm{Au}-\mathrm{PEG}$ conjugates with a different number of PEG molecules attached per particle were purified on a desalting column before using them for the SEC, FCS, and thermophoresis experiments. From gel electrophoresis, the mobilities $m$ of all conjugates were then transformed in corresponding effective diameters $d_{\text {eff }}$ by using a mobilitydiameter calibration curve created with phosphine-stabilized $\mathrm{Au}$ nanoparticles $^{20}$ (SI §III):

$$
\begin{aligned}
& d_{\text {eff, } 1 \%}(m)=-85.0 \cdot \ln \left[\left(m / m_{10 \mathrm{~nm}, 1 \%}\right) / 1.05\right]+6[\mathrm{~nm}] \\
& d_{\mathrm{eff}, 2 \%}(m)=-37.7 \cdot \ln \left[\left(m / m_{10 \mathrm{~nm}, 2 \%}\right) / 1.12\right]+6[\mathrm{~nm}] \quad(\text { Formula } 1)
\end{aligned}
$$

Here, $m / m_{10 \mathrm{~nm}, y}$ refers to the electrophoretic mobility of the conjugates $(\mathrm{m})$ in relation to the mobility of $10 \mathrm{~nm}$ phosphinestabilized $\mathrm{Au}$ particles $\left(m_{10 \mathrm{~nm}, y}\right)$ that have been run on a gel with the same agarose concentration $y(y=1 \%$ or $2 \%) .{ }^{19,20}$
Size Exclusion Chromatography. $\mathrm{CdSe} / \mathrm{ZnS}$ and $\mathrm{Au}$ particles saturated with PEG molecules of different molecular weight were run on different high-performance liquid chromatography (HPLC) size exclusion columns, and the elution volume $v_{\mathrm{e}}$ (i.e., the total volume of the mobile phase when the particles come out of the column) of the particles was measured (similarly, elution times $t_{\mathrm{e}}$, i.e., the time after which the particle fraction is eluted from the column, could have been measured) (see Figure 2) (SI §IV). In order to normalize the data to one universal curve, the elution volumes $v_{\mathrm{e}}$ were transformed into partition coefficients $K_{\mathrm{SEC}}{ }^{52,53}$ The partition coefficient corresponds to the fraction of accessible pore volume for sample particles and can obtain values $0 \leq K_{\mathrm{SEC}} \leq 1$. Very small particles or molecules can occupy the total pore volume (as they are small enough to fully penetrate the pores of the gel), and $K_{\text {SEC }}=1$. Very large particles are totally excluded from the pore volume, and $K_{\mathrm{SEC}}=0$. In this way, the $K_{\mathrm{SEC}}$ value is a measure of the size of the particles: the smaller and larger the particles are, the closer their $K_{\mathrm{SEC}}$ values come to 1 and 0 , respectively. In contrast to elution volumes or elution times, the $K_{\text {SEC }}$ values are normalized quantities and thus do not depend in first order on experimental parameters such as sample volume, flow rate, or column geometry (SI §IV):

$$
\begin{aligned}
K_{\mathrm{SEC}}(\text { particle })= & {\left[\left(t_{\mathrm{e}}(\text { particle })-t_{\mathrm{e}}(\text { biggest particle })\right] /\right.} \\
& {\left[\left(t_{\mathrm{e}}(\text { smallest particle })-t_{\mathrm{e}}(\text { biggest particle })\right]\right.} \\
= & {\left[\left(v_{\mathrm{e}}(\text { particle })-v_{\mathrm{e}}(\text { biggest particle })\right] /\right.} \\
& {\left[\left(v_{\mathrm{e}}(\text { smallest particle })-v_{\mathrm{e}}(\text { biggest particle })\right]\right.} \\
= & {\left[\left(v_{\mathrm{e}}(\text { particle })-v_{0}\right] /\left[v_{\mathrm{t}}-v_{0}\right] \quad(\text { Formula })\right.}
\end{aligned}
$$

The elution volume of the very small particles is referred to as the total liquid volume $v_{\mathrm{t}}$, and the elution volume of the very large particles is referred to as dead or void volume $v_{0}$. In order to experimentally obtain $v_{\mathrm{t}}$ and $v_{0}$, aceton (a very small particle) and $\lambda$-DNA (a very big particle) were run, and their elution volumes $\left(=v_{\mathrm{t}}\right.$ and $\left.v_{0}\right)$ were measured. For generating a calibration curve that relates partition coefficients $K_{\mathrm{SEC}}$ to

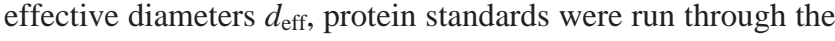
columns, and their elution volumes were determined from the elution peak maxima and converted into partition coefficients. The size $d_{\text {eff }}$ of each protein standard was estimated as 2 times the hydrodynamic radius of the protein. ${ }^{53}$ By plotting the partition coefficients of different proteins versus their effective diameter and extrapolating these data, a calibration curve $d_{\mathrm{eff}}{ }^{-}$ $\left(K_{\mathrm{SEC}}\right)$ was obtained (see Figure 2 ). By using this calibration curve, the partition coefficients derived for the $\mathrm{CdSe} / \mathrm{ZnS}-\mathrm{PEG}$ and $\mathrm{Au}-\mathrm{PEG}$ conjugates could be converted into effective diameters. As for the gel electrophoresis experiments, the effective diameters are obtained by a comparison with standard samples of known diameter. In the case of the gel electrophoresis experiments, phosphine-stabilized Au nanoparticles were used. These particles could not be used for the SEC measurements, as they got stuck in the columns. Therefore a series of globular proteins had to be used as standard samples for the SEC measurements.

Fluorescence Correlation Spectroscopy. Samples of CdSe/ $\mathrm{ZnS}-\mathrm{PEG}$ conjugates were mounted on an Axiovert200 confocal microscope with a $40 \times$ water immersion objective (CApochromat, NA $=1.2$ ) and a ConfoCor 2 FCS module (whole setup: Zeiss, Germany). Fluorescence was excited with the $488 \mathrm{~nm}$ line of an Ar ion laser. The focal volume was calibrated with Alexa488 (diffusion coefficient $D=316 \mu \mathrm{m}^{2} \mathrm{~s}^{-1}$, Molecular Probes). The nanocrystals were diluted to a concentration 
TABLE 2: Effective Diameters $d_{\text {eff }}[\mathrm{nm}]$ of CdSe/ZnS-PEG Conjugates

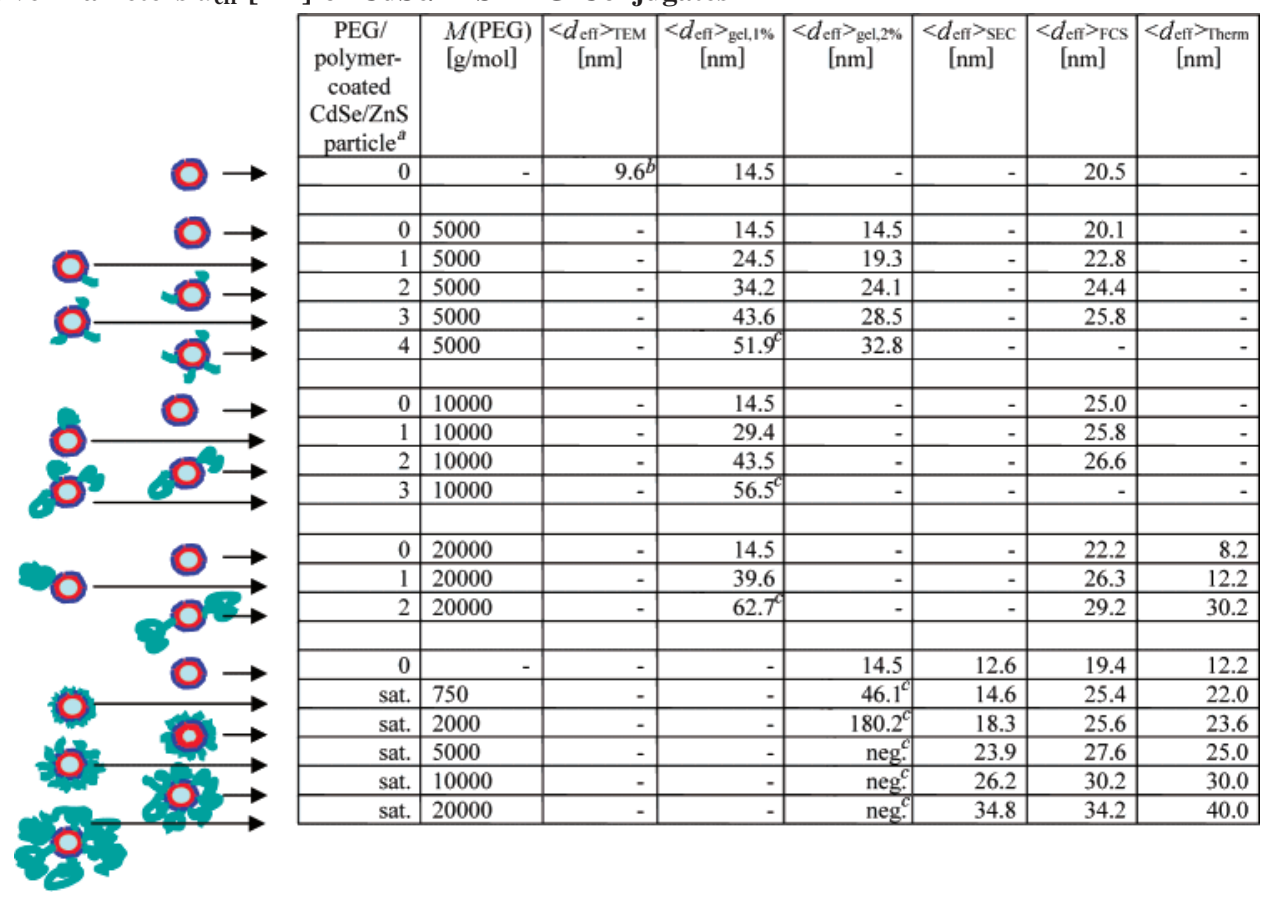

${ }^{a}$ PEG molecules (drawn in green) of different molecular weight $M_{\mathrm{w}}$ have been attached to the surface of polymer-coated CdSe/ZnS particles, as already sketched in Table $1 .{ }^{14}$ Either zero, one, two, three, or as many as possible ("sat.") PEG molecules were attached per particle, and the effective particle diameter $<d_{\text {eff }}>$ was measured with TEM, gel electrophoresis ("gel", $1 \%$ and $2 \%$ agarose concentration), FCS, SEC, and thermophoresis ("Therm"). The particles with single PEGs attached per particle (first 13 samples) had been separated with gel electrophoresis with subsequent extraction from the gel and purification before their diameters were measured with SEC, FCS, and thermophoresis. ${ }^{b}$ This value has to be considered as approximation. ${ }^{c}$ These values are not realistic (as they are either too big or negative) due to limitations of the applied technique (see SI §II.2).

TABLE 3: Effective Diameters $d_{\text {eff }}[\mathrm{nm}]$ of Au-PEG Conjugates

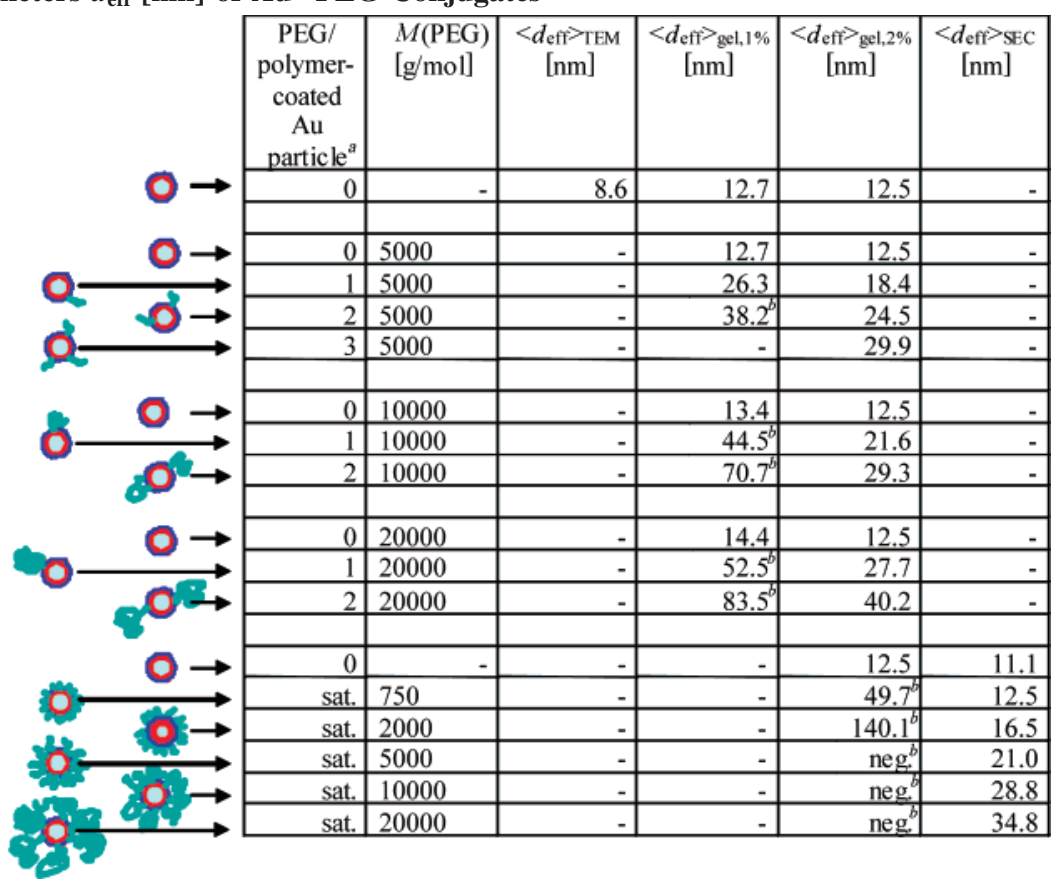

${ }^{a}$ PEG molecules of different molecular weight $M_{\mathrm{w}}$ have been attached to the surface of polymer-coated Au particles. ${ }^{14}$ Either zero, one, two, three, or as many as possible ("sat.") PEG molecules were attached per particle, and the effective particle diameter $\left\langle d_{\text {eff }}\right\rangle$ was measured with gel electrophoresis ("gel", $1 \%$ and $2 \%$ agarose concentration) and SEC. ${ }^{b}$ These values cannot be taken into account (as they are too big or negative) and demonstrate the limitations of gel electrophoresis for size measurements (see SI §II.2).

of $\sim 10 \mathrm{nM}$, which corresponds to roughly one particle per focal volume. Time traces of the fluorescence intensity were recorded with an avalanche photodiode with single-photon sensitivity. From the fluorescence intensity traces, autocorrelation functions were calculated (see Figure 3) (SI §V). By fitting the experimentally obtained autocorrelation functions with model functions for freely diffusing particles, the diffusion coefficients of the particles were obtained as fit parameters. ${ }^{37-39,54}$ The diffusion 
TABLE 4: Effective Diameters $\boldsymbol{d}_{\text {eff }}$ of Commercially Available Quantum Dots ${ }^{a, b}$

\begin{tabular}{lcccc}
\hline \multicolumn{1}{c}{ sample } & $\begin{array}{c}\left.<d_{\text {eff }}\right\rangle_{\text {gel, } 2 \%} \\
{[\mathrm{~nm}]}\end{array}$ & $\begin{array}{c}\left.<d_{\text {eff }}\right\rangle_{\text {SEC }} \\
{[\mathrm{nm}]}\end{array}$ & $\begin{array}{c}\left.<d_{\text {eff }}\right\rangle_{\text {Therm }} \\
{[\mathrm{nm}]}\end{array}$ & $\begin{array}{c}\left.<d_{\text {eff }}\right\rangle_{\text {mean-value }} \\
{[\mathrm{nm}]}\end{array}$ \\
\hline QD655 carboxyl & 16.6 & 11.8 & 14.0 & $14.1 \pm 2.4^{d}$ \\
QD655 SA & $133.0^{c}$ & 20.5 & 25.6 & $23.1 \pm 1.6$
\end{tabular}

${ }^{a}$ Quantum Dot Corporation, $655 \mathrm{~nm}$ emission, polymer shell with carboxyl groups and with additional streptavidin (SA) modification (Qdot655 ITK carboxyl, \#2132-1, and Qdot655 streptavidin conjugate, \#1012-01). ${ }^{b}$ The values were obtained with gel electrophoresis (2\% agarose gels), SEC (column with Sephadex S-400), and thermophoresis. ${ }^{c}$ This value cannot be taken into account due to charge effects (see SI $\S$ II.2). ${ }^{d}$ This value is in the same range as the diameter obtained from Pons et $\mathrm{al}^{24}$ for particles with slightly smaller cores. a)

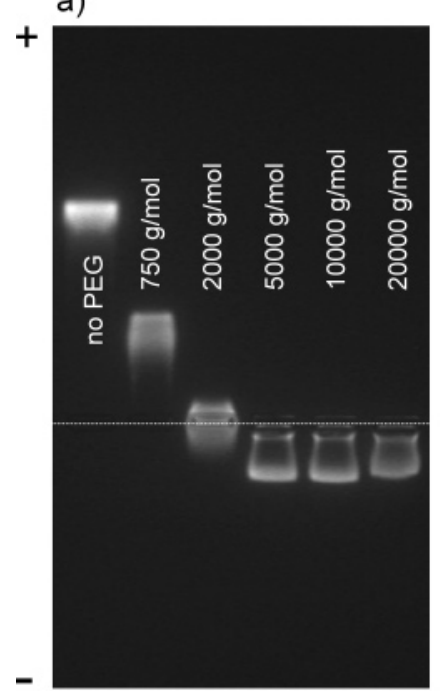

b)

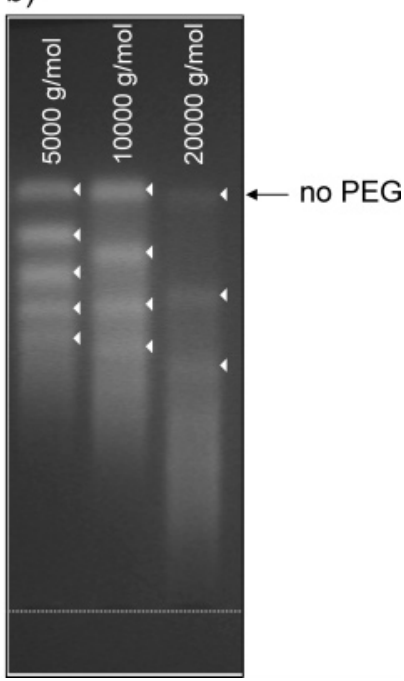

Figure 1. 1. Gel electrophoresis for polymer-coated $\mathrm{CdSe} / \mathrm{ZnS}$ nanoparticles. The "+" and "-" symbols indicate the direction of the applied electric field, and the dashed line marks the position where the samples have been loaded into the wells of the gel. (a) $2 \%$ agarose gel with particles with no PEG and particles whose surface has been saturated with PEG of 750, 2000, 5000, 10000, and 20000 Da molecular weight. (b) $1 \%$ agarose gel of particles to whose surface only a few PEG molecules of different molecular weight have been bound. The particles with no, one, two, and so forth PEG molecules attached per particle yield discrete bands on the gel.

coefficients were then converted into effective diameters by using the Stokes-Einstein relation. In contrast to the gel electrophoresis and HPLC measurements, the effective diameters obtained with FCS measurements are absolute values and do not depend on a direct comparison with standard samples of known diameter. However, the setup has to be calibrated with a dye of known diffusion constant for all measurements. Since the $\mathrm{Au}-\mathrm{PEG}$ conjugates do not fluoresce, they could not be analyzed with FCS.

Thermophoresis. Strong local temperature gradients were used to manipulate concentration patterns in solution, all optically. Figure $4 \mathrm{a}$ shows the typical time course of an experiment. A solution of nanoparticles was continuously monitored by fluorescence microscopy, and the local fluorescence was used as measure for the particle concentration. An infrared laser was then used to introduce an inhomogeneous radial symmetric concentration pattern by inducing thermophoretic motion. After a few seconds, when the concentration in the center of the heat spot had decreased to at least $90 \%$ of the initial condition, the heating source was turned off. The temperature relaxed nearly instantaneously and was followed by the much slower flattening of the concentration profile by diffusion. These time-resolved concentration profile data were compared with data obtained in a one-dimensional (1D) radial simulation over time (Figure 4b). By comparison of the experimental and simulated data, the mean diffusion coefficient of the fluorescent particles in solution was obtained, and from this the hydrodynamic diameter of the particles was derived using the Stokes-Einstein relation (SI §VI). In contrast to the gel electrophoresis and HPLC measurements the effective diameters obtained with thermophoresis measurements do not depend on a comparison with standard samples of known diameter, but are absolute values. Since the $\mathrm{Au}-\mathrm{PEG}$ conjugates do not fluoresce, they could not be analyzed with thermophoresis.

PEG Radius. The increase in size of particles with a PEG shell should correspond to the dimensions of the PEG molecules. The effective diameters of free PEG molecules can be calculated from Formula 3, which was obtained by SEC, ${ }^{55}$ whereby $r_{\mathrm{h}}$ is the hydrodynamic radius, and $M_{\mathrm{w}}$ is the molecular weight of the PEG:

$$
d_{\mathrm{eff}, \mathrm{PEG}}=2 r_{\mathrm{h}}=0.03824 M_{\mathrm{w}}^{0.559}
$$

(Formula 3)

\section{Results and Discussion}

Transmission Electron Microscopy. The results for the TEM analysis on particles without PEG modification are summarized in Table 1 . However, these values have to be considered as very rough estimates with only limited reliability (SI §II.2). First of all, measurements had to be performed on dried samples. While, in solution, the hydrophobic surfactant chains repel each other, on a TEM grid, the particles can come so close to each other that the surfactant chains intercalate. More severe, the size distribution after the polymer coating, that is, the size-distribution of the entire system inorganic core plus organic shell, is by far not as good as that of the original particle solution, and the particles therefore do not assemble anymore nicely into two-dimensional lattices. Therefore, the particle-toparticle distance curves are smeared out, and the derived effective particle diameters have to be interpreted with care. As the particles have to be measured in the dried state, the effective diameters as determined by TEM do not contain any interaction with the solvent (as, for example, a cloud of counterions). Therefore, the values obtained for the effective diameter for $\mathrm{CdSe} / \mathrm{ZnS}$ as well as Au particles with TEM are significantly smaller than the effective diameters determined with methods in which the particles are dispersed in their solvents (see first lines of Tables 2 and 3).

Gel Electrophoresis. In Figure 1, examples for particles separated by gel electrophoresis are shown. As with SEC, not only can the particle diameter be estimated, but the particles can also be sorted and fractionated by size in small preparative scale. In comparison to SEC, the size resolution of gel electrophoresis is significantly better, as particles with zero, one, two, and so forth PEG molecules can be clearly separated by gel electrophoresis, but not with the columns used for SEC. However, there are severe limitations for the determination of effective diameters. The particles need to possess a very high colloidal stability in the electrolytic solution, which is needed to drive the current, otherwise they agglomerate and get stuck on the gel. The biggest problem, however, is obtaining an appropriate calibration curve that relates electrophoretic mobility to effective size. As electrophoretic mobility depends on both size and charge, any calibration curve for size can only be valid for objects of similar charge. Furthermore, the physical properties such as stiffness and flexibility of the particles used to obtain 


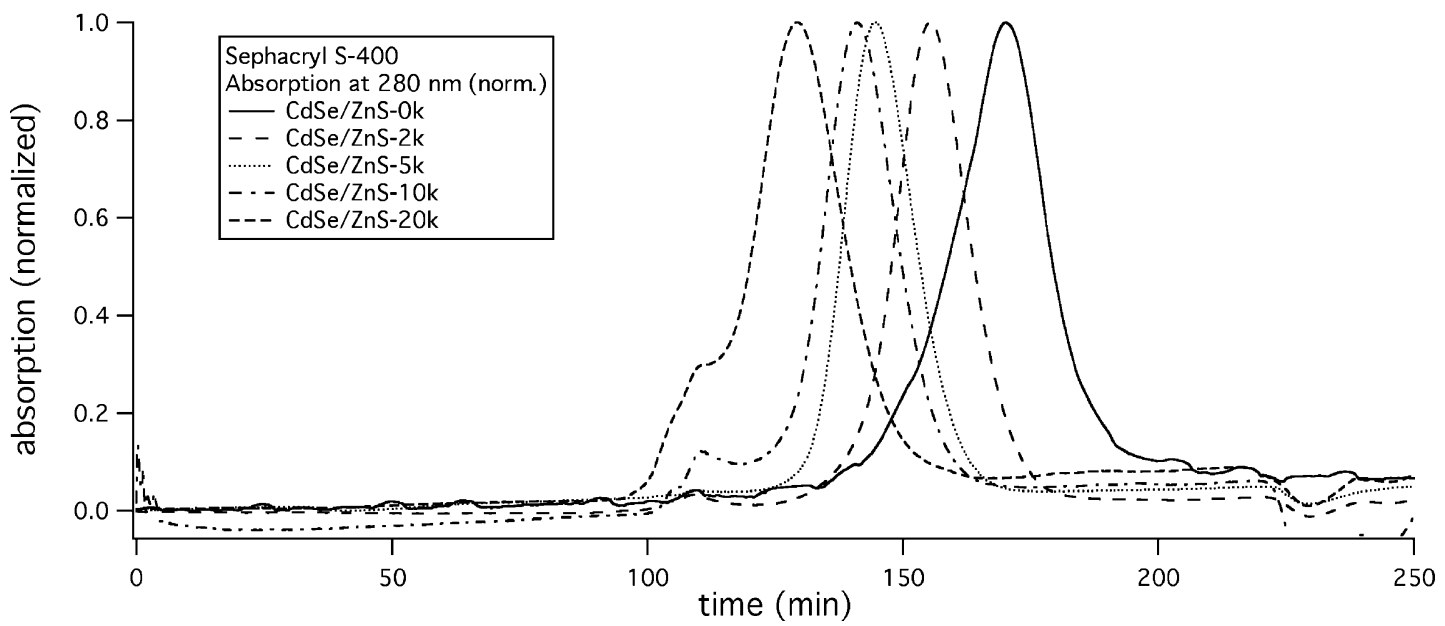

Figure 2. Size exclusion chromatograph (SEC) with a Sephacryl S-400 filled column of CdSe/ZnS nanoparticles whose surface is saturated with PEG molecules of different molecular weight (no PEG, $2 \mathrm{kDa}, 5 \mathrm{kDa}, 10 \mathrm{kDa}, 20 \mathrm{kDa}$ ). Plotted is the absorption of the eluted solution versus the elution time. The bigger the particles are, due to the attachment of PEG of higher molecular weight, the earlier they are eluted from the column.

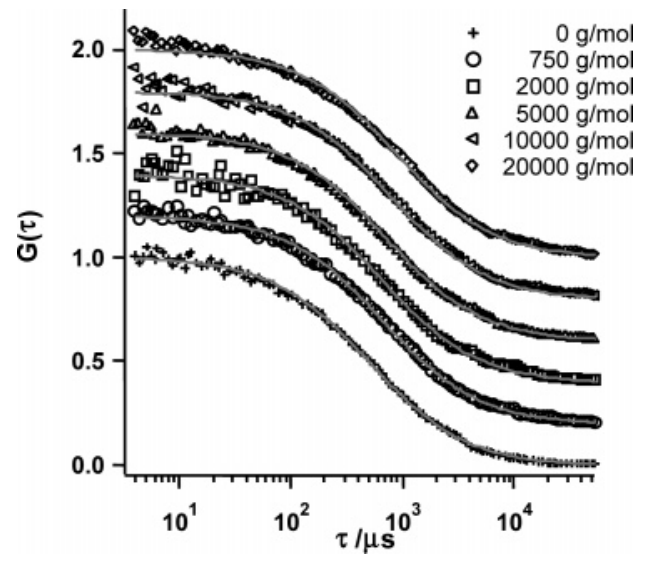

Figure 3. Autocorrelation functions $G(\tau)$ obtained from the FCS data for polymer-coated $\mathrm{CdSe} / \mathrm{ZnS}$ particles whose surface is saturated with PEG molecules of different molecular weight $(750 \mathrm{~g} / \mathrm{mol}, 2 \mathrm{~kg} / \mathrm{mol}$, $5 \mathrm{~kg} / \mathrm{mol}, 10 \mathrm{~kg} / \mathrm{mol}, 20 \mathrm{~kg} / \mathrm{mol}$ ). The curves for the respective particles are shifted along the $y$-axis for the purpose of better visualization. The particles with no PEG attached are referred to as particles with PEG of molecular weight 0 (bottom graph). The experimental data were fitted with an analytical function (shown as gray lines), which yielded the effective diffusion coefficient as one fit parameter (see SI §V).

the calibration curve must be similar to those of the particles that are to be investigated. Basically, two different types of particles could be used in order to obtain calibration curves: biological macromolecules or colloidal nanoparticles. We have tried oligonucleotides ${ }^{19,20}$ as well as proteins for calibration, but both yielded very different mobility values compared to the ones obtained for colloidal nanoparticles. As linear flexible molecules, oligonucleotides can move in a different way through the pores of a gel compared to rigid inorganic colloidal nanoparticles. On the other hand, the surface charge density of oligonucleotides is, in first order, constant, therefore oligonucleotides are sorted by size and not by charge. In contrast to average oligonucleotides, proteins possess a secondary and tertiary structure and can be thought of in crude approximation as elastic spherical particles. However, proteins can have different surface charge densities comprising the full spectra from negative to neutral to positive. Because it is hard to find a set of proteins with different sizes but with the same charge density as the particles that are to be investigated, proteins are also not suited as standard particles for obtaining a calibration curve that relates electrophoretic mobilities to size. Therefore we have chosen inorganic colloidal nanoparticles of different size but with identical
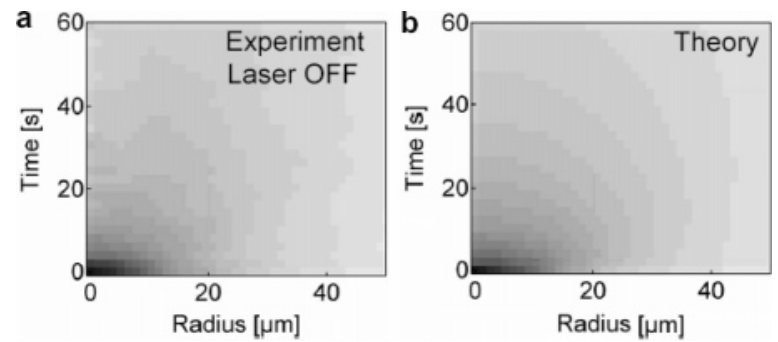

Figure 4. Thermophoresis and back-diffusion of nanometer-sized particles in solution. Thermodiffusion is used to form a concentration gradient in solution by heating a micrometer-sized spot. (a) (experiment) The graph shows the development of radial concentration averages over time after the heating laser is switched off (black: low concentration; gray: high concentration). As can be seen from the plot, the concentration inhomogeneity relaxes within $60 \mathrm{~s}$. The data shown correspond to a particle size of approximately $30 \mathrm{~nm}$ in diameter. (b) (theory) The diffusion constant $D$ is obtained by performing 1D finite element simulations with $D$ as a free parameter, until the theory in b matches the experiment in a.

negatively charged surface coatings (bis( $p$-sulfonatophenyl)phenylphosphine dihydrate) as size standards. There are known problems for these standards as well. First, there is an inherent uncertainty in the standard, as the effective hydrodynamic diameter is not known and is assumed to be the hard core $\mathrm{Au}$ particle diameter plus 2 times the length of the phosphine molecules, without taking into account the cloud of counterions. ${ }^{20}$ However, comparison with the literature data obtained from dynamic light scattering shows that they are in good agreement with the assumed values. Second, attached macromolecules (such as $\mathrm{DNA}^{20}$ ) form a soft shell around the hard inorganic core, which can be compressed by the gel. This can be seen in the data of Tables 2 and 3: the effective diameters derived from $1 \%$ agarose gels are always larger than the ones from $2 \%$ agarose gels, as the soft shell is compressed more on gels of higher percentage. This difference in data obtained with gels of different percentages has already been observed with DNA-modified sulfonate-stabilized Au particles. ${ }^{20}$ Even in this case where there is only a minor influence of charge on the electrophoretic mobilities, the diameters obtained with gels of different percentages differed significantly, whereby the differences were most significant for long DNA strands and particles with a lot of attached DNA (= thick soft shells). In this study, PEG molecules were used for particle modification. As has already been reported, ${ }^{14}$ attachment of sufficient amounts of PEG to negatively charged polymer-coated particles can reverse 
the polarity of the particles to a positive net charge, so that they migrate toward the negative electrode during gel electrophoresis (see Figure 1). This effect might be due to positive ions adsorbed to the PEG. ${ }^{56}$ At any rate, this charge effect makes the determination of effective diameters of PEGylated particles with gel electrophoresis almost impossible. Due to the charge effect, the derived diameters are too large, and, for particles saturated with long PEG, no diameter can be derived due to the change in polarity (see Tables 2 and 3). The more PEG is attached per particle and the higher the molecular weight of the PEG, the more unreliable the results become. Although, in our experience, gel electrophoresis is the most sensitive of the here-described methods to resolve changes in size (e.g., the attachment of a single molecule), reliable absolute numbers of effective diameters can be only derived under very restricted conditions when the charge density of the sample to be investigated is highly similar to that of the particles used as size standards.

Size Exclusion Chromatography. Analogous to gel electrophoresis, SEC is not only an analytical method, but particles of different diameter can be fractionated on a preparative scale. Here, sorting by size is also achieved by a porous matrix, but, in contrast to gel electrophoresis where particles are driven by an electric current and small particles run faster, in SEC smaller particles are retarded because they can access a larger pore volume of the column packing. For all the columns used in this study, the resolution by size was lower for SEC compared to gel electrophoresis, since, with SEC, particles with zero, one, two, three, and so forth PEG molecules bound per particle could never be separated, while this was easily achieved with gel electrophoresis. Size measurements with both methods rely on appropriate size standards. In SEC, charge effects, that is, electrostatic interaction of charged particles with charges in the gel, are typically reduced by a mobile phase with a high salt concentration that screens residual charges of the column material. Unfortunately, this rules out the use of the sulfonatestabilized Au particles that were used for gel electrophoresis as size standards, as these particles tend to agglomerate at high salt concentrations and get stuck in the columns. We therefore used globular proteins as size standards, as we could exclude charge effects as in the case of gel electrophoresis. Good estimates for effective hydrodynamic diameters exist for many proteins. On the other hand, the size range of available proteins is limited. We were unable to find spherically shaped (globular) proteins with a diameter as large as the largest of our PEGmodified nanoparticles (ca. $40 \mathrm{~nm}$ ). The derived effective diameters for PEGylated nanoparticles from the extrapolated calibration curve are therefore more reliable the smaller the particles are (see Tables 2 and 3 and Figure 2).

Fluorescence Correlation Spectroscopy. Unlike gel electrophoresis or SEC, FCS is a purely analytical method and does not allow for separation and subsequent collection of fractions of particles with different diameters. The effective diameters derived from the FCS measurements (see Table 2 and Figure 3) are consistent with our previous findings ${ }^{11,41}$ and also consistent within themselves. The more and the longer PEG is added to the particles, the bigger the measured effective diameters are. Since FCS is based on single-particle experiments, each effective diameter reported in Table 2 corresponds to the mean value of the diameters of hundreds of particles from each sample. Within one sample series (i.e., different PEGs have been attached to the same batch of polymer-coated particles and the measurements were performed directly after each other with exactly the same FCS setup conditions), even the attachment of single PEG molecules could be resolved, that is, the resolution limit is better than that for the increase in diameters for particles upon attachment of zero, one, two, and three PEGs per particle (see Table 2). However, the absolute values of effective diameters that have been recorded on the same type of particles but under different experimental conditions (i.e., when the particles were extracted from different gels before the measurements, when the FCS setup was recalibrated, etc.) vary significantly. As an example, we take the mean diameters for plain polymer-coated particles (i.e., zero PEGs per particle) from the different series from Table 2 . The mean effective diameter and standard deviation of the five different samples $\left(<d_{\mathrm{eff}, \mathrm{FCS}}>\right.$ $=<20.5 \mathrm{~nm}, 20.1 \mathrm{~nm}, 25.0 \mathrm{~nm}, 22.2 \mathrm{~nm}, 19.4 \mathrm{~nm}>$ ) is $22.0 \pm$ $2.8 \mathrm{~nm}$. The standard deviation has to be seen as an error bar for absolute measurements. The error bar is in the same range as the increase in the particle diameter upon the attachment of individual PEG molecules per particle. We therefore conclude that, although relative changes in the effective particle diameter upon the attachment of molecules within the same batch of particles and under the same setup conditions can be resolved with good precision, there is a significant error of about $3 \mathrm{~nm}$ in the determination of absolute effective diameters. There are two main sources for systematic errors in deriving absolute effective diameters: (i) Although FCS does not need a size standard, the focal volume has to be calibrated for each set of measurements with a dye molecule of known diffusion constant and thus known hydrodynamic diameter. Each error of the calibration is propagated to the results of the following measurements. (ii) In contrast to organic fluorophores, colloidal quantum dots exhibit no exponential triplet state decay but rather blinking behavior on all time-scales, which influences the recorded florescence intensity time traces. To our knowledge, so far, no analytical expression has been derived to account for this fact. ${ }^{11,41,42}$

Thermophoresis. The values obtained by thermophoresis increase as expected with the molecular weight of the covalently coupled PEG molecules. The method measures the mean diffusion coefficients of the whole ensemble. Species without or with less surface modification would, in principle, lead to a higher overall diffusion coefficient and smaller radius, respectively. The sizes obtained for nanoparticles saturated with PEG molecules are listed in Table 2, and a typical experiment is shown in Figure 4. In contrast to gel electrophoresis and SEC, no calibration curve of size standards is needed to obtain the effective diameters. Although thermophoresis, like FCS, is based on obtaining effective diameters by measuring the diffusion of the dispersed particles, the values obtained with both methods differ significantly (see Table 2). The standard deviation in the measurements between similar samples is in the same range as that with FCS (polymer-coated $\mathrm{CdSe} / \mathrm{ZnS}$ with no PEG: $<d_{\text {eff,Therm }}>=<8.2 \mathrm{~nm}, 12.2 \mathrm{~nm}>=10.2 \pm 2.8 \mathrm{~nm}$; see last column of Table 2; the deviations within one method are attributed to variations in the sample, e.g., due to differences in the gel extraction procedure). The difference in the effective diameter of about a factor of $2\left(<d_{\text {eff,Therm }}\right\rangle \approx$ $10 \mathrm{~nm} ;\left\langle d_{\mathrm{eff}, \mathrm{FCS}}>\approx 22 \mathrm{~nm}\right.$ ) for plain polymer-coated nanoparticles as determined with thermophoresis and FCS can therefore not be explained by the resolution limit due to sample variations $(\approx 3 \mathrm{~nm})$ of both methods. Although we cannot explain the origin of this discrepancy, we speculate that it might arise from the different particle concentrations used for the measurements. Whereas FCS is a single-molecule-based method and thus requires extremely diluted particle solutions, thermophoresis is an ensemble-based method, and typically more 
TABLE 5: Polymer-Coated Au and CdSe/ZnSe Nanoparticles Coated with Saturated Layers of PEG Molecules of Different Molecular Weight $M_{\mathrm{w}}{ }^{a}$

\begin{tabular}{|c|c|c|c|c|c|c|c|c|c|c|}
\hline $\begin{array}{c}M_{\mathrm{W}}(\mathrm{PEG}) \\
{[\mathrm{g} / \mathrm{mol}]}\end{array}$ & $\begin{array}{c}<d_{\mathrm{eff}}>_{\mathrm{SEC}} \\
{[\mathrm{nm}]} \\
\text { Au core }\end{array}$ & $\begin{array}{c}<d_{\text {eff }}>_{\text {SEC }} \\
{[\mathrm{nm}]} \\
\text { CdSe/ZnS } \\
\text { core }\end{array}$ & $\begin{array}{c}<d_{\mathrm{eff}}>_{\mathrm{FCS}} \\
{[\mathrm{nm}]} \\
\mathrm{CdSe} / \mathrm{ZnS} \\
\text { core }\end{array}$ & $\begin{array}{c}<d_{\text {eff }}>_{\text {Therm }} \\
{[\mathrm{nm}]} \\
\text { CdSe/ZnS } \\
\text { core }\end{array}$ & $\begin{array}{c}1 / 2 \Delta d_{\mathrm{eff}, \mathrm{SEC}} \\
{[\mathrm{nm}]} \\
\text { Au core }\end{array}$ & $\begin{array}{c}1 / 2 \Delta d_{\mathrm{eff}, \mathrm{SEC}} \\
{[\mathrm{nm}]} \\
\mathrm{CdSe} / \mathrm{ZnS} \\
\text { core }\end{array}$ & $\begin{array}{c}1 / 2 \Delta d_{\mathrm{eff}, \mathrm{FCS}} \\
{[\mathrm{nm}]} \\
\mathrm{CdSe} / \mathrm{ZnS} \\
\text { core }\end{array}$ & $\begin{array}{c}1 / 2 \Delta d_{\text {eff, Therm }} \\
{[\mathrm{nm}]} \\
\text { Au core }\end{array}$ & $\begin{array}{c}1 / 2<\Delta d_{\mathrm{eff}}> \\
{[\mathrm{nm}]}\end{array}$ & $\begin{array}{c}d_{\mathrm{eff}, \mathrm{PEG}} \\
{[\mathrm{nm}]}\end{array}$ \\
\hline 0 & 11.1 & 12.6 & 19.4 & 12.2 & & & & & & \\
\hline 750 & 12.5 & 14.6 & 25.4 & 22.0 & 0.7 & 1.0 & 3.0 & 4.9 & $2.4 \pm 2.0$ & 1.5 \\
\hline 2000 & 16.5 & 18.3 & 25.6 & 23.6 & 2.7 & 2.9 & 3.1 & 5.7 & $3.6 \pm 1.4$ & 2.7 \\
\hline 5000 & 21.0 & 23.9 & 27.6 & 25.0 & 5.0 & 5.7 & 4.1 & 6.4 & $5.3 \pm 1.0$ & 4.5 \\
\hline 10000 & 28.8 & 26.2 & 30.2 & 30.0 & 8.9 & 6.8 & 5.4 & 8.9 & $7.5 \pm 1.7$ & 6.6 \\
\hline 20000 & 34.8 & 34.8 & 34.2 & 40.0 & 11.9 & 11.1 & 7.4 & 13.9 & $11.1 \pm 2.7$ & 9.7 \\
\hline
\end{tabular}

${ }^{a}$ The first line hereby corresponds to plain polymer-coated nanoparticles without PEG modification. In columns $2-5$, the mean effective diameters $<d_{\text {eff }}>$ of the particles as determined with different methods are listed. These values originate from the data in Tables 2 and 3. The thickness of the PEG layers of different molecular weight around polymer-coated particles are derived as half of the difference of the total diameters of the PEG-coated and the plain polymer-coated particles: $1 / 2 \Delta d_{\mathrm{eff}}\left(M_{\mathrm{w}}(\mathrm{PEG})=\mathrm{X}\right)=\left(<d_{\mathrm{eff}}\left(M_{\mathrm{w}}(\mathrm{PEG})=\mathrm{X}\right)>-<d_{\mathrm{eff}}\left(M_{\mathrm{w}}(\mathrm{PEG})=0\right)>\right) / 2$. These values are listed in columns 6-9. In column 10, the mean thickness of the PEG layers (as the average value of the different methods) $1 / 2 \Delta d_{\mathrm{eff}}$ is given. The last column shows the diameters of free PEG molecules as calculated with Formula 3.55

concentrated particle solutions are used in order to obtain signals with sufficient intensity. Particle-particle interaction plays a different role under both conditions. Also the statistical effect of a certain amount of aggregated particles is different. We estimate the relative concentration of aggregates to contribute less than linearly to the diffusion coefficient measured by thermophoresis, since larger particles are depleted more strongly and the back diffusion is slower. Thus, the measured signal stems mostly from single-particle diffusion. The role of particle interactions will be analyzed in future experiments under optimized conditions with particle concentrations of less than $10 \mathrm{nM}$. This would allow direct comparison with FCS experiments. The values obtained with SEC in ensemble measurements of relatively concentrated particle solutions for plain polymercoated particles correspond more to the values obtained with thermophoresis than those obtained with FCS, which also gives some indication about the importance of the particle concentration used for the measurements.

Thickness of Organic Coating Layers. In the following, we focus on the polymer-coated particles according to our own procedure (Tables 2 and 3). The mean value of all our different methods for the effective diameters of polymer-coated $\mathrm{CdSe} /$ $\mathrm{ZnS}$ is $<d_{\text {eff,gel }},\left(d_{\mathrm{eff}, \mathrm{FCS}}\right), d_{\mathrm{eff}, \mathrm{SEC}}, d_{\mathrm{eff}, \mathrm{Therm}}>=<14.4,12.6$, (19.5), $12.2>=13.1 \pm 1.2 \mathrm{~nm}(14.7 \pm 3.4 \mathrm{~nm}$ with the value obtained with FCS), and, for Au nanoparticles, it is $<d_{\text {eff,gel }}$, $d_{\text {eff,SEC }}, d_{\text {eff,Therm }}>=<12.5,11.6>=12.0 \pm 0.6 \mathrm{~nm}$. The hard core diameter as determined by TEM is $5.3 \mathrm{~nm}$ for $\mathrm{CdSe} / \mathrm{ZnS}$ and $4.6 \mathrm{~nm}$ for the Au nanoparticles. This leads to an effective thickness of the organic shell of $(13.1-5.3) \mathrm{nm} / 2=3.9 \mathrm{~nm}$ in the case of $\mathrm{CdSe} / \mathrm{ZnS}$ and $(12.0-4.6) \mathrm{nm} / 2=3.7 \mathrm{~nm}$ in the case of $\mathrm{Au}$. Besides the values obtained with FCS, the values derived with the other techniques correspond well, and we conclude that, after polymer coating, the effective thickness of the organic layer around the inorganic particle core is around $3.5-4.0 \mathrm{~nm}$. Whereas this value for the plain polymer-coated particles seems quite reliable, the uncertainties in absolute size determination get higher the larger the molecules attached to the polymer shell are. The addition of a saturated layer of $20 \mathrm{kDa}$ PEG molecules to the polymer surface increases the thickness of the organic layer by $<\Delta d_{\text {eff,gel }}, \Delta d_{\text {eff,FCS }}$, $\Delta d_{\mathrm{eff}, \mathrm{SEC}}>/ 2=<(34.8-12.6),(34.2-19.4),(40.0-12.2)>$ $\mathrm{nm} / 2=10.8 \pm 3.3 \mathrm{~nm}$ in the case of the $\mathrm{CdSe} / \mathrm{ZnS}$ particles.

In Table 5, the thickness of the saturated layer of the PEG molecules bound to the particle surface is compared with the effective diameter of free PEG molecules as determined by Formula 3, whereby the thickness of the PEG layer was calculated as half of the difference in diameter of the PEG- coated and plain polymer-coated particles. All obtained values for the PEG molecules bound to the nanoparticles are slightly larger than those of free PEG molecules. This finding can be explained by a more stretched configuration of the random coil of the PEG molecules when they are attached by one end to the densely occupied surface of a saturated nanoparticle, compared to the presumably more symmetric configuration of PEG molecules in free solution. In any case, the agreement demonstrates that relative increments in particle size can be determined with much higher accuracy than absolute diameters.

\section{Conclusions}

The total particle diameter can be estimated by the core diameter plus 2 times the thickness of the organic layer, which is, for simple coatings, the length of the surfactant molecule. While this eventually works well for short molecules when the length of these molecules is small compared to the particle, it becomes more complicated for longer and more complex molecules or even complex polymer (multi)layers. Here the size depends strongly on assumptions about the steric configuration of the molecules on the curved nanoparticle surface. Furthermore, the effective hydrodynamic diameter also depends on hydration: interaction of the particles with the solvent results in larger effective sizes, ${ }^{57}$ even in the most simple case where the stabilizer molecules form a monolayer around the inorganic core. Although several studies exist in which the effective diameters of particles have been measured, most of these studies are either based on only one method or only one type of particle surface. ${ }^{24,58,59}$ A more detailed discussion can be found in the Supporting Information.

The more molecules are attached and thus the bigger the particles become, the more unreliable size measurements are. First, the hybrid nature of the particles with a rigid inner inorganic core and a soft organic shell becomes more pronounced, which eventually leads to problems for the methods in which the measurements take place in a matrix that can compress the particles. Especially, a random coil of a linear polymer such as PEG can be easily deformed depending on the technique used for the size determination. Second, for the methods using calibration with size standards, there is the problem of a lack of appropriate size standards of sufficient size. Third, the charge composition can also change (in particular, if positively charged molecules are attached), which leads to the failure of gel electrophoresis but might also affect the other methods.

Thus, depending on the actual particle nature but also on the intended application, the adequate method for measurement has 
to be chosen with great care. For instance, when particles are designed to enter into pores, the diameter determined by gel electrophoresis or SEC might be better suited than the one determined by free diffusion.

Different methods to measure effective sizes of colloidal nanoparticles are based on different physical principles, resulting in deviations of the resulting particle diameters between the different methods. This finding is not surprising and points to a general problem and uncertainty: although within one measurement effective diameters can be determined in a consistent way with relatively small errors, bigger discrepancies arise between values obtained with different methods. This implies that the comparison with control samples (e.g., before and after a certain conjugation step) remains indispensable and that all derived absolute numbers for nanoparticle diameters have to be considered with care.

Acknowledgment. The authors would like to thank Andrea Rauh for help with the analysis of the TEM images and Dr. Fritz Simmel and Prof. Dr. Joachim Rädler for helpful discussion. The authors are grateful to Prof. Dr. Joachim Rädler for providing the FCS setup and expertise of his institute. Quantum dots with organic and streptavidin coating were a gift from Quantum Dots Corp. The project was funded by the Deutsche Forschungsgemeinschaft (DFG Emmy Noether Grant) and the European Union (STREP NanoInteract grant). M.Z. is grateful for a fellowship from the International Doctorate Program NanoBio-Technology at the Center for Nanoscience.

Supporting Information Available: Details of particle synthesis, TEM analysis, gel electrophoresis, SEC, FCS, and thermophoresis experiments, and a comparison of the different methods. This material is available free of charge via the Internet at http://pubs.acs.org.

\section{References and Notes}

(1) Scher, E. C.; Manna, L.; Alivisatos, A. P. Philos. Trans. R. Soc. London, Ser. A 2002, 361, 241.

(2) Kumar, S.; Nann, T. Small 2006, 2, 316.

(3) Kudera, S.; Carbone, L.; Zanella, M.; Cingolani, R.; Parak, W. J.; Manna, L. Phys. Status Solidi C 2006, 203, 1329.

(4) Templeton, A. C.; Wuelfing, W. P.; Murray, R. W. Acc. Chem. Res. 2000, 33, 27.

(5) Michalet, X.; Pinaud, F. F.; Bentolila, L. A.; Tsay, J. M.; Doose, S.; Li, J. J.; Sundaresan, G.; Wu, A. M.; Gambhir, S. S.; Weiss, S. Science 2005, 307, 538.

(6) Pellegrino, T.; Kudera, S.; Liedl, T.; Javier, A. M.; Manna, L.; Parak, W. J. Small 2005, 1, 48

(7) Fischer, C.-H.; Lilie, J.; Weller, H.; Katsikas, L.; Henglein, A. Ber. Bunsen-Ges. Phys. Chem. 1989, 93, 61.

(8) Fischer, C.-H.; Weller, H.; Katsikas, L.; Henglein, A. Langmuir 1989, $5,429$.

(9) Eychmüller, A.; Katsikas, L.; Weller, H. Langmuir 1990, 6, 1605 (10) Wu, M. X.; Liu, H.; Liu, J.; Haley, K. N.; Treadway, J. A.; Larson, J. P.; Ge, N.; Peale, F.; Bruchez, M. P. Nat. Biotechnol. 2003, $21,41$.

(11) Pellegrino, T.; Manna, L.; Kudera, S.; Liedl, T.; Koktysh, D.; Rogach, A. L.; Keller, S.; Rädler, J.; Natile, G.; Parak, W. J. Nano Lett. 2004, 4, 703.

(12) Luccardini, C.; Tribet, C.; Vial, F.; Marchi-Artzner, V.; Dahan, M. Langmuir 2006, 22, 2304.

(13) Nann, T. Chem. Commun. 2005, 2005, 1735.

(14) Sperling, R. A.; Pellegrino, T.; Li, J. K.; Chang, W. H.; Parak, W. J. Adv. Funct. Mater. 2006, 16, 943.

(15) Zanchet, D.; Moreno, M. S.; Ugarte, D. Phys. Rev. Lett. 1999, 82, 5277.

(16) Fink, J.; Kiely, C. J.; Bethell, D.; Schiffrin, D. J. Chem. Mater. 1998, 10, 922. 2291.

(17) Yonezawa, T.; Onoue, S.-y.; Kimizuka, N. Langmuir 2001, 17,

(18) Zanchet, D.; Micheel, C. M.; Parak, W. J.; Gerion, D.; Alivisatos, A. P. Nano Lett. 2001, 1, 32.

(19) Parak, W. J.; Pellegrino, T.; Micheel, C. M.; Gerion, D.; Williams, S. C.; Alivisatos, A. P. Nano Lett. 2003, 3, 33.

(20) Pellegrino, T.; Sperling, R. A.; Alivisatos, A. P.; Parak, W. J. J. Biomed. Biotechnol., submitted for publication, 2007.

(21) Pinaud, F.; King, D.; Moore, H.-P.; Weiss, S. J. Am. Chem. Soc. 2004, 126, 6115 .

(22) Song, X.; Li, L.; Qian, H.; Fang, N.; Ren, J. Electrophoresis 2006, $27,1341$.

(23) Nehilla, B. J.; Vu, T. Q.; Desai, T. A. J. Phys. Chem. B 2005, 109, 20724.

(24) Pons, T.; Uyeda, H. T.; Medintz, I. L.; Mattoussi, H. J. Phys. Chem. B 2006, 110, 20308.

(25) Wilcoxon, J. P.; Provencio, P. P. J. Phys. Chem. B 2005, 109 , 13461

(26) Siebrands, T.; Giersig, M.; Mulvaney, P.; Fischer, C.-H. Langmuir 1993, 9, 2297.

(27) Fischer, C.-H.; Giersig, M.; Siebrands, T. J. Chromatogr., A 1994, 670,89 .

(28) Fischer, C.-H.; Kenndler, E. J. Chromatogr., A 1997, 773, 179.

(29) Wei, G.-T.; Liu, F.-K. J. Chromatogr., A 1999, 836, 253.

(30) Wei, G.-T.; Liu, F.-K.; Wang, C. R. C. Anal. Chem. 1999, 71, 2085

(31) Jimenez, V. L.; Leopold, M. C.; Mazzitelli, C.; Jorgenson, J. W.; Murray, R. W. Anal. Chem. 2003, 75, 199.

(32) Al-Somali, A. M.; Krueger, K. M.; Falkner, J. C.; Colvin, V. L. Anal. Chem. 2004, 76, 5903.

(33) Krueger, K. M.; Al-Somali, A. M.; Falkner, J. C.; Colvin, V. L. Anal. Chem. 2005, 77, 3511.

(34) Kuga, S. J. Chromatogr., A 1981, 206, 449

(35) Holtzhauer, M.; Rudolph, M. J. Chromatogr., A 1992, 605, 193.

(36) Magde, D.; Elson, E.; Webb, W. W. Phys. Rev. Lett. 1972, 29 705.

(37) Eigen, M.; Rigler, R. Proc. Natl. Acad. Sci. U.S.A. 1994, 91, 5740.

(38) Schwille, P.; Bieschke, J.; Oehlenschläger, F. Biophys. Chem. 1997, $66,211$.

(39) Krichevsky, O.; Bonnet, G. Rep. Prog. Phys. 2002, 65, 251.

(40) Zhang, P.; Li, L.; Dong, C.; Qian, H.; Ren, J. Anal. Chim. Acta 2005, 546, 46.

(41) Liedl, T.; Keller, S.; Simmel, F. C.; Rädler, J. O.; Parak, W. J. Small 2005, 1, 997.

(42) Doose, S.; Tsay, J. M.; Pinaud, F.; Weiss, S. Anal. Chem. 2005, 77, 2235.

(43) Dong, C.; Bi, R.; Qian, H.; Li, L.; Ren, J. Small 2006, 2, 534.

(44) Jin, T.; Fujii, F.; Yamada, E.; Nodasaka, Y.; Kinjo, M. J. Am. Chem. Soc. 2006, 128, 9288 .

(45) Duhr, S.; Arduini, S.; Braun, D. Eur. Phys. J. E 2004, 15, 277.

(46) Duhr, S.; Braun, D. Phys. Rev. Lett. 2006, 96, 168301.

(47) Duhr, S.; Braun, D. Proc. Natl. Acad. Sci. U.S.A. 2006, 103, 19678.

(48) Reiss, P.; Bleuse, J.; Pron, A. Nano Lett. 2002, 2, 781.

(49) Dabbousi, B. O.; Rodriguez-Viejo, J.; Mikulec, F. V.; Heine, J. R.; Mattoussi, H.; Ober, R.; Jensen, K. F.; Bawendi, M. G. J. Phys. Chem. B 1997, 101, 9463.

(50) Yu, W. W.; Qu, L.; Guo, W.; Peng, X. Chem. Mater. 2003, 15 , 2854.

(51) Brust, M.; Walker, M.; Bethell, D.; Schiffrin, D. J.; Whyman, R. J. Chem. Soc., Chem. Commun. 1994, 801.

(52) Mori, S.; Barth, H. G. Size Exclusion Chromatography; Springer: New York, 1999.

(53) Hagel, L. Gel Filtration. In Protein Purification. Principles, High Resolution Methods and Applications, 2nd ed.; Janson, J.-C., Ryden, L., Eds.; John Wiley \& Sons: New York, 1998.

(54) Amediek, A.; Haustein, E.; Scherfeld, D.; Schwille, P. Single Mol. 2002, 3, 201.

(55) Fee, C. J.; Alstine, J. M. V. Bioconjugate Chem. 2004, 15, 1304.

(56) Sartori, R.; Sepulveda, L.; Quina, F.; Lissi, E.; Abuin, E. Macromolecules 1990, 23, 3878.

(57) Xu, X.; Rosi, N. L.; Wang, Y.; Huo, F.; Mirkin, C. A. J. Am. Chem. Soc. 2006, 128, 9286

(58) Smith, A. M.; Duan, H.; Rhyner, M. N.; Ruan, G.; Nie, S. Phys. Chem. Chem. Phys. 2006, 8, 3895.

(59) Yu, W. W.; Chang, E.; Falkner, J. C.; Zhang, J.; Al-Somali, A. M.; Sayes, C. M.; Johns, J.; Drezek, R.; Colvin, V. L. J. Am. Chem. Soc. 2007, 129, 2871. 